\title{
Vergleich von Eye Movement Desensitization and Reprocessing (EMDR) und Biofeedback/ Stress-Impfungstraining in der Behandlung von Prüfungsangst
}

\author{
Rosemary Cook-Vienot \\ Raymond J. Taylor \\ Counseling Services of Houston, Texas
}

\begin{abstract}
EMDR und Biofeedback / Stress-Impfungstraining und Nicht-Behandlung (NB) wurden in ihrer Wirkung, Prüfungsangst zu reduzieren, verglichen. Hierzu wurden jeder Kohorte 30 College-Studenten mit hoher Prüfungsangst randomisiert zugewiesen. Prä / Post-Bewertungen wurden unter Verwendung des Test Anxiety Inventory (TAI), State-Trait Anxiety Inventory (STAI), Rational Behaviour Inventory (RBI) und Autonomic Perception Questionnaire (APQ) durchgeführt. Behandelnde Therapeuten waren qualifizierte Fachleute mit mindestens zweijähriger Berufserfahrung in ihrem jeweiligen Verfahren. Die statistische Analyse, in der eine wechselseitige Varianzanalyse mit wiederholten Messungen durchgeführt wurde, ergab für alle Meßverfahren außer dem RBI signifikante Zusammenhänge zwischen dem Zeitfaktor (d.h. den Prä- / Post-Messungen) und den eingesetzten Verfahren. Die Veränderungs-Messwerte wurden post hoc Newman-Keuls Analysen unterzogen und zeigten, dass sowohl EMDR als auch B / SIT die Prüfungsangst signifikant reduzierten. Insgesamt erzielte EMDR hierbei bessere Ergebnisse als B / SIT.
\end{abstract}

Schlüsselwörter: Prüfungsangst; EMDR; Biofeedback; Stress-Impfung

$\mathbf{P}$ rüfungsangst ist ein Problem, dem Menschen während ihrer Lebenszeit häufig ausgesetzt sind. Oftmals sind sie damit konfrontiert, an irgendeiner Form der Wissensbewertung teilnehmen zu müssen. Menschen, die an Prüfungsangst leiden, tendieren dazu, evaluative Situationen als persönliche Bedrohung wahrzunehmen. So sind sie in Test-Situationen oft übermäßig nervös, angespannt, besorgt und emotional aufgewühlt. Sie neigen außerdem zu negativen Selbstüberzeugungen, die ihre Aufmerksamkeit ablenken und ihre Konzentration während der Prüfungen beeinträchtigen (Beidel, Turner, \& Dancu, 1985; Schulz, Alpers, \& Hoffmann, 2008). Dies kann zu überwältigender Angst führen.

Prüfungsangst kann in vielen unterschiedlichen Situationen auftreten. Beispiele hierfür sind unter anderem: Nicht zur höheren Schule zugelassen zu werden aufgrund von unzureichenden Ergebnissen, das NichtBestehen der Prüfung zum Wirtschaftsprüfer, schlechtes Abschneiden bei Prüfungen an der medizinischen Fakultät, Durchfallen bei der Anwaltsprüfung oder nicht in der Lage zu sein, einen bestimmte für den College-Abschluss erforderlichen Kurs zu bestehen. Prüfungen müssen ebenfalls für den Führerschein abgelegt werden oder um in der Armee aufgenommen zu werden, oder wann immer ein Beleg der Leistungsfähigkeit gefordert ist. Die Mehrzahl der Klienten gibt an, sich vorbereitet zu haben und den Stoff sicher zu beherrschen, jedoch in der tatsächlichen Prüfungssituation „sei der Kopf leer“ oder sie erleben eine "Angst-Attacke", die es ihnen unmöglich macht, die Prüfungsaufgaben zu erledigen. Üblicherweise wurde Prüfungsangst als unwichtig abgetan und Klienten berichten, dass ihnen angeraten

This article originally appeared as Cook-Vienot, R., \& Taylor, R. J. (2012). Comparison of Eye Movement Desensitization and Reprocessing and Biofeedback/Stress Inoculation Training in Treating Test Anxiety. Journal of EMDR Practice and Research, 6(2), 62-72. Translated by Marlen Lenzen. 
wurde, sich einfach anderen Dingen zuzuwenden, ihre angestrebten Ziele zu vergessen und zu akzeptieren, dass sie nun einmal keine guten Prüfungsabsolventen sind.

Prüfungsangst existiert kulturübergreifend. Hierzu liegen Studien aus Israel, Japan, China, den Niederlanden, Deutschland, Belgien, der Türkei, England und den Vereinigten Staaten vor. Prüfungsangst betrifft ebenso beide Geschlechter, sämtliche Altersgruppen und ist unter allen sozialökonomischen Voraussetzungen anzutreffen.

Forschungsbefunde im Bereich der Prüfungsangst zeigen, dass sie sich ebenso aus physiologischen wie aus kognitiven Bestandteilen zusammensetzt (Cassady \& Johnson, 2002; Eckman \& Shean, 1997). Die Behandlungsnotwendigkeit von Prüfungangst ist offensichtlich. In Untersuchungen von NavehBenjamin, Lavi McKeachie und Lin (1997) wurde ein Negativ-Zusammenhang zwischen der Reduzierung von Prüfungsangst und der Merkfähigkeit für Lernmaterial gefunden. Pluess, Conrad und Wilhelm (2009) stellen fest, dass die typische Genesungsrate für eine Angsterkrankung bei Anwendung herkömmlicher Therapieverfahren bei nur ungefähr $40 \%$ liegt. Aufgrund dessensindBehandlungen, denen einbesseres Verständnis potentieller psychophysiologischer Mechanismen zugrunde liegt, von entscheidender Bedeutung. Falsetti (2003) stellte fest, dass die Behandlung von Trauma- und Angstsymptomen durch die Modifikation von kognitiver Verhaltenstherapie (zum Beispiel mit Stress-ImpfungsTherapie) verbessert wurde.

\section{Eye Movement Desensitization and Reprocessing (EMDR)}

Ursprünglich eingeführt von Shapiro (2001) ist Eye Movement Desensitization and Reprocessing (EMDR) ein international anerkanntes Verfahren zur wirksamen Behandlung von Posttraumatischen Belastungsstörungen (z.B. American Psychiatric Association, 2004; Bisson \& Andrew, 2007 / 2009; Bradley, Greene, Russ, Dutra, \& Western, 2005; U.S. Department of Veterans Affairs \& U.S. Department of Defense, 2004 / 2010). Es gab ca. 20 randomisierte kontrollierte Studien, in denen die Behandlung mit EMDR bei Posttraumatischen Belastungsstörungen untersucht wurde. Rothbaum et al. (2005) und van der Kolk et al. (2007) verglichen EMDR und kognitive Verhaltenstherapie und Carlson, Chemtob, Rusnak, Hedlund und Muraoka (1998) verglichen EMDR mit der Biofeedback-Methode, wobei sich EMDR in beiden Fällen als effektiver erwies. Lee, Gavriel, Drummond,
Richards und Greenwald (2002) verglichen EMDR mit der Stress-Impfungs-Behandlung (bei verlängerter Exposition). In der Katamnese zeigten sich keine signifikanten Unterschiede, jedoch in der Nachuntersuchung schnitt EMDR besser ab.

Im Hinblick auf die Behandlung verschiedener Angststörungen wurde EMDR viel weniger untersucht und diesbezügliche Befunde weisen uneinheitliche Ergebnisse auf. De Jongh und ten Broeke (2009) äuBerten die Vermutung, dass EMDR höchst wirksam sein könnte im Zusammenhang mit Angststörungen, die ihren Ursprung in einem traumatischen Erlebnis haben.

EMDR (Shapiro, 2001) fokussiert auf die Desensibilisierung und das Reprozessieren schmerzlicher Erinnerungen und thematisiert vergangene Erlebnisse, gegenwärtige Auslöser und zukünftige, potentielle Herausforderungen. Shapiro postulierte, dass die Reduktion oder Auflösung des Leidens unter aufwühlenden Erinnerungen letztlich zur Linderung der gegenwärtigen Symptome, einer verbesserten Sichtweise des eigenen Selbst, zur Entlastung von Körperstörungen und der Bewältigung von gegenwärtigen und zukünftigen Auslösern führen wird.

In einer EMDR-Sitzung konzentriert sich der Patient auf die Erinnerung und das damit assoziierte affektive, kognitive und somatische Material während kurzer (ca. 24-sekündiger) Sequenzen schneller, rhythmischer, horizontaler Augenbewegungen, die unter der Anleitung des Therapeuten durchgeführt werden. Nach jeder Sequenz teilt der Patient dem Therapeuten mit, welches Material assoziiert wurde. Dieses wird dann üblicherweise zum Gegenstand der nächsten Behandlungseinheit. Der Patient bewertet den Angstlevel, indem er eine 10-Punkte-Skala (Subjective Unit of Disturbance / SUD) verwendet, wobei 0 neutrale Intensität und 10 die höchstmögliche Belastung oder Angst repräsentiert (Shapiro, 2001; Wolpe, 1991). Nach der Desensibilisierung der Zielerinnerung wird eine Verankerung der positiven Kognition vorgenommen, unter Einsatz derselben Augenbewegungen. Anschließend bewertet der Klient die Ergebnisse dieses Prozesses anhand einer 7-Punkte-Skala (VOC, Stimmigkeit der Kognition), von $1=$ absolut falsch bis zu 7 = absolut wahr, um den Erfolg des Prozesses einzuschätzen (Shapiro, 2001). Das Durcharbeiten der Erinnerung wird als abgeschlossen betrachtet, wenn der Patient keine Belastung mehr auf der SUD-Skala angibt, die positive Kognition als valide eingeschätzt wird, kein somatischer Stress mehr angegeben wird und alle gegenwärtigen und zukünftigen Auslöser behandelt wurden. 


\section{Die Behandlung von Prüfungsangst durch EMDR}

EMDR hat sich als effektiv erwiesen in der Behandlung von Prüfungsangst, sowohl bei einstündiger als auch bei zweistündiger Behandlungsdosis. Maxfield und Melnyk (2000) verglichen EMDR-Behandlung und Nicht-Behandlung ${ }^{1}$, und zeigten unter Einsatz des Test Anxiety Inventory (TAI) und des State-Trait Anxiety Inventory (STAI), dass eine einzige EMDRSitzung signifikant die emotionalen und physiologischen Komponenten von Prüfungsangst reduzierte. Hierbei verbesserten sich die Teilnehmer von der 90. Perzentile (TAI, vor Behandlungsbeginn) zur 50. Perzentile nach Behandlungsende. In einer kontrollierten Studie mit 62 Undegraduate-Studenten zeigten Stevens und Florell (1999), dass EMDR im Vergleich zu Rational-Emotiver Therapie (RET) und Information-Only Condition ${ }^{2}$ am effektivsten die Belastung reduzierte, wohingegen RET generalisierte Testangst besser reduzierte als die Information-Only Condition.

Andere Studien haben uneindeutige Resultate ergeben. Baumann und Melnyk (1994) verglichen eine EMDR-Variante mit metronomisch gesteuertem (metronome-timed) Fingerklopfen und fanden heraus, dass beide Therapien einen Abfall auf der Emotionalitäts-Subskala des TAI bewirkten, jedoch nicht auf der Besorgnis- (worry) oder auf der Gesamt-TAI-Skala. Gosselin und Matthews (1995) verglichen EMDR mit Augenbewegungen und EMDR mit fixiertem Blick sowohl bei geringer als auch hoher Versagenserwartung. Sie fanden heraus, dass alle Teilnehmer signifikante Verbesserungen auf der TAI-Skala zeigten und dass kein weiterer Effekt erkennbar war, weder in Bezug auf die angewandte EMDR-Mechanik (Abw./fixiert), noch für hohe / niedrige Versagenserwartung. Enright, Baldo und Wykes (2000) äußerten die Notwendigkeit nach weiterer komparativer Forschung betreffend EMDR und anderer anerkannter Verfahren zur Behandlung von Prüfungsangst.

\section{Biofeedback}

Biofeedback ist ein weit verbreitetes Verfahren zur Behandlung physiologischer Symptome bei Stress und Angst. Die Ursprünge des Biofeedback gründen in der Kernannahme der Lerntheorie: den Prinzipien des operanten Konditionierens . Die Forschung hat die Wirksamkeit von Biofeedback bei der Reduzierung der emotionalen oder physiologischen Komponenten der Prüfungsangst bestätigt (Hurwitz, Kahane, \& Mathieson, 1986; Ratanasiripong, Sverduk, Hayashino, \& Prince, 2010). Schwartz und Andrasik (2003) zeigten, dass Biofeedback am effektivsten ist, wenn es mit kognitiver Verhaltenstherapie kombiniert wird.

Während der Behandlung von Stress- oder Angstzuständen durch Biofeedback lernt der Klient, wie er oder sie die physiologischen Reaktionen kontrollieren kann, die bei belastenden Gedankengängen auftreten. Diesbeinhaltet viele Techniken, wiezumBeispielMuskelRelaxation, Handerwärmung und Zwerchfellatmung. Während der Biofeedback-Behandlung wird der Klient zunächst an das Kontrollieren von Stress oder Angst herangeführt, während er an einen Monitor angeschlossen ist, der alle oder einige der folgenden Messungen abbildet: Körpertemperatur, Galvanische Hautreaktion(GSR), die die autonome nervöse Aktivität misst, oder Elektromyographie (EMG), welche die Muskelspannung misst (Schwartz \& Andrasik, 2003). Mit der Behandlung soll der Patient letztlich befähigt werden, auch ohne das Monitor-Feedback in alltäglichen Lebenssituationen die gleichen Ergebnisse zu replizieren. Indem der Klient lernt, Kontrolle über diese Reaktionen zu gewinnen, ist er in der Lage, deren ängstliche Aufladung zu reduzieren und erwartet ein besseres Abschneiden in der Prüfungssituation.

\section{Die Behandlung von Prüfungsangst durch Biofeedback}

In einigen Studien wurde die Wirkung der Biofeedback-Methode im Zusammenhang mit Prüfungsangst und ihren verschiedenen Komponenten untersucht. Mit Biofeedback wurde die Komponente der emotionalen oder physiologischen Erregung, sowie die kognitive bzw. Besorgnis-Komponente von Prüfungsangst erfolgreich behandelt (Kiselica, Baker, Thomas, \& Reedy, 1994). Ratanasiripong et al. (2010) demonstrierten den effektiven Gebrauch einer tragbaren Biofeedback-Vorrichtung zur Behandlung von Prüfungsangst bei College-Studenten.

Die Forschung bestätigt, dass Biofeedback die physiologischen Symptome von Prüfungsangst wirksam reduziert. Jedoch wird zur Behandlung des Besorgnis- oder kognitiven Aspektes der zusätzliche Einsatz einer kognitiven Modifikations-Behandlung angeraten (Kiselica et al., 1994). Verschiedene Studien haben Biofeedback mit anderen Verfahren zur Behandlung von Prüfungsangst verglichen. Obwohl all diese Untersuchungen einen positiven Effekt von Biofeedback auf Prüfungsangst belegten, wurde bei keiner von ihnen deutlich, dass Biofeedback signifikant effektiver ist als eines der Vergleichsverfahren. Biofeedback wurde mit Hypnose (Spies, 1979), systematischer Desensibilisierung (Romano \& Cabianca, 
1978) und mit Auslösereiz-kombinierter Relaxation (Counts, Hollandsworth, \& Alcorn, 1978) verglichen.

\section{Stress-Impfungstraining}

Stress-Impfung ist eine Art selbst-angeleitetes Training, das viele verschiedene Coping-Skills kombiniert, die in der Behandlung von Prüfungsangst anwendbar sind. Meichenbaum (1996) hat diesen Ansatz mit dem Immunisierungs-Ansatz in der Medizin verglichen: Wenn ein Mensch geimpft wird, wird ihm die Möglichkeit gegeben, in einer kontrollierten Umgebung eine kleine Dosis des stress-bezogenen Stimulus zu bewältigen. Die Lernerfahrung, mit kleinen Einheiten des Stimulus umzugehen, hilft, Coping-Skills für bedrohlichere Situationen zu entwickeln (Rehm \& Rokke, 1988). Darauf zugeschnitten, Menschen in belastenden Situationen durch Stärkung und Entwicklung ihrer Coping-Strategien zu unterstützen, gliedert sich die Stress-Impfung in drei Phasen (Meichenbaum, 1996; Sheehy \& Horan, 2004): Die erste Phase hat edukativen Charakter. Um dem Klienten Grundlagenwissen zum Verständnis seiner eigenen Reaktionen zu vermitteln, wird er in ein bis zwei theoretische Ansätze zur Stress-Reaktion eingeführt. Um einen höchstmöglichen therapeutischen Erfolg beim Implementieren der Coping-Strategien erzielen zu können ist es wichtig, dass die Informationen im Erfahrungskontext des Klienten leicht anwendbar sind. Zum Beispiel könnte der Klient die Informationen erhalten, dass Angst-Reaktionen aus zwei Haupt-Komponenten bestehen, nämlich aus physiologischer und kognitiver Erregung (Bilder und Selbst-Überzeugungen) und dass diese Komponenten zur Verstärkung der Angst beitragen.

Die zweite Phase beinhaltet die Einübung vieler verschiedener Coping-Techniken, die für jeden Klienten individuell entwickelt werden, wie zum Beispiel die Formulierung positiver Selbst-Überzeugungen, das Imaginieren einer angenehmen Szene oder die Anwendung anderer Entspannungs-Techniken. Die letzte Phase schließlich besteht darin, dass der Klient die Coping-Techniken in einer gesteigerten Staffelung von Stress-Situationen anwendet. Dieser Prozess kann während der Therapie-Sitzungen beginnen und wird ausgeweitet, indem der Klient seine CopingTechniken in Alltagssituationen und in anderen mäßig belastenden Situationen anwendet.

\section{Die Behandlung von Prüfungsangst durch Stress-Impfungstraining}

Saunders, Driskell, Johnston und Salas (1996) führten eine Auswertung vorliegenden Studien zur Behand- lung von Prüfungsangst und anderen Leistungsproblemen durch Stress-Impfungstraining durch und kamen dabei zu dem Ergebnis, dass sich dieser Ansatz in neun Studien als effektiv - mit mäßigen bis deutlichen Ergebnissen - erwiesen hatte. Sheehy und Horan (2004) fanden heraus, dass Stress-Impfungstraining bei Jura-Studenten im ersten Studienjahr wirksam bei der Behandlung von Prüfungs- und Studienangst war (unter Verwendung von Meichenbaums (1996) dreistufiger Methode).

\section{Methode}

\section{Ziel}

Das Ziel dieser Studie war es, EMDR mit der Biofeedback-Methode und dem Stress-Impfungstraining zu vergleichen. Die Wirksamkeit jeder der drei Behandlungsmethoden wird durch die Forschung gestützt. Außerdem ermöglicht Biofeedback eine objektive Messung physiologischer Erregung. Das Stress-Impfungstraining beinhaltet eine Komponente, die kognitive Restrukturierung ermöglicht. Dadurch eignete es sich innerhalb dieser Studie für die Kombination mit Biofeedback. Die Null-Hypothese war, dass es keinen Unterschied in den Behandlungsergebnisse von EMDR und Biofeedback, bzw. StressImpfungstraining, geben würde.

\section{Teilnehmer}

Dreißig erwachsene Teilnehmer (College-Studenten von örtlichen Junior- und 4-Year Undergraduate Colleges und Graduate Schools) im Alter von 19 bis 53 Jahren ( $\mathrm{M}=36,9)$ nahmen an der Studie teil. Acht Teilnehmer waren männlich und 22 waren weiblich. Ihre Ausbildungszeit bewegte sich zwischen 8 bis 18 Jahren $(\mathrm{M}=14,4)$. Psychologische Ausschluss-Kriterien beinhalteten aktiven Drogen-Missbrauch, dissoziative Störungen, Psychosen und andere psychologische Probleme außer Prüfungsangst, die auf der Symptom Checkliste-90-R als hochrangig eingestuft werden. Medizinische Ausschluss-Kriterien beinhalteten Sehstörungen, Epilepsie, neurologische Einschränkungen und Schwangerschaft. Andere Screening-Kriterien beinhalteten die Selbsteinschätzung bzgl. subjektivem Belastungsgrad durch Prüfungsangst, gemessen anhand der SUD-Skala. Probanden mit einem SUDWert über 6 wurden der Studie zugewiesen.

\section{Bewertung}

\section{Prescreening-Messinstrumente}

Mit potenziellen Teilnehmern wurde die SymptomCheckliste-90-R und die Dissociative Events Scale 
durchgeführt, um psychologische Probleme zusätzlich zur Prüfungsangst feststellen zu können.

\section{Prä-, Post- und Follow-Up Messungen}

Die Testungen wurden vor Behandlungsbeginn durchgeführt und 30-45 Tage nach dem Vortest und / oder dem Behandlungsabschluss. Zur Einschätzung vor Behandlungsbeginn und nach Behandlungsabschluss kamen folgende Skalen zum Einsatz: TAI, STAI, Rational Behavior Inventory (RBI) und Autonomic Perception Questionnaire (APQ). Dem Testleiter wurden die Ergebnisse der psychologischen Testungen bis zum Abschluss der Untersuchung nicht mitgeteilt.

Test Anxiety Inventory. Das TAI wurde von Spielberger (1980) entwickelt und besteht aus einem 20 Items umfassenden Selbsteinschätzungs -Fragenkatalog, wobei die Antworten mit der 4-Punkte-Skala von Likert erfasst werden. Die Testungs-Ergebnisse bewegen sich auf einer Skala von 20 bis 80 Punkten. Je höher die Punktzahl, desto höher das Angstlevel. Die anschließende Auswertung ergibt drei Punktwerte: Eine Gesamtpunktzahl, den Punktwert für, Besorgnis ' und den für, Emotion`.

State-Trait Anxiety Inventory. Das STAI (Spielberger, 1983) wurde oft zur Messung von State- und Trait-Angst ${ }^{3}$ bei der Erforschung von Prüfungsangst verwendet (Reed et al., 1980; Thyer et al., 1981). Es besteht aus einem 20 Items umfassenden Fragenkatalog, dessen Antworten mit der 4-Punkte-Skala von Likert erfasst werden. Die Testungs-Ergebnisse umfassen eine Skala von 20 bis 80 Punkten. Je höher die Punktzahl, desto höher die Angst. Die Ergebnisse des STAI können bei der Erstellung einer klinischen Diagnose verwendet werden; hier sind sie bei der Differentialdiagnose zwischen Angst und Depression hilfreich. Ebenso können sie hilfreich sein in der psychologischen- und Gesundheitsforschung, sowie für die Diagnosestellung hinsichtlich pathologischer Angst bei Klienten in medizinischen, chirurgischen und psychiatrischen Kontexten (Spielberger, 2008).

Rational Behavioral Inventory. Das RBI (Shorkey \& Whiteman, 1977) wurde zur quantitativen Bestimmung irrationaler Überzeugungen in der RET entwickelt. Es wurde ebenfalls eingesetzt, um die Veränderung selbstbezogener Kognitionen zu messen (Thyer et al., 1981). Es besteht aus einem 37 Items umfassenden Fragebogen, wobei höhere Punktzahl höhere Rationalität der Überzeugungen anzeigt.

Autonomic Perception Questionnaire. Das APQ (Borkovec, 1978) wurde entwickelt, um physiologische Erregung zu messen. Die Angst-bezogene
Variante des APQ besteht aus einem Selbsteinschätzungsinventar und misst die Aufmerksamkeit für Körperreaktionen in Angst-auslösenden Situationen (zum Beispiel: „Wenn ich Angst habe, kann ich spüren, dass mein Herz schneller schlägt"). Im APQ wird, unter Verwendung einer 21 Items umfassenden 9-Punkte-Likert-Skala, vom Probanden die Kurzbeschreibung einer Situation erfragt, in welcher der Proband Angst empfand. Die Erstbeschreibung des Probanden dient hierbei als Referenz, um die Reaktionen einzustufen. Abschließend werden die Ergebnisse zu einer Gesamtpunktzahl zusammengefasst, die sich zwischen 21 und 180 bewegt. Eine höhere Gesamtpunktzahl bedeutet in diesem Zusammenhang höhere wahrgenommene autonome Aktivität (zum Beispiel Hyperventilation, schwitzende Hände).

\section{Behandlung}

Die Teilnehmer wurden randomisiert entweder der EMDR-, B / SIT- oder Nicht-Behandlungs-Kohorte (NB) zugewiesen. Die Behandlung wurde sowohl für EMDR und B / SIT lege artis standardisiert.

Beide Gruppen (B / SIT, EMDR) erhielten dieselbe Anzahl von Therapie-Minuten; diese wurden jedoch unterschiedlich zwischen den Sitzungen aufgeteilt. B / SIT-Teilnehmer erhielten drei 90-minütige Sitzungen, die in erster Linie für die Behandlung verwendet wurden. Die Sitzungen der EMDR-Teilnehmer waren aufgeteilt in eine 45-minütige Sitzung zur Vermittlung einführender und therapeutischer Informationen; darauf folgten dann drei 75 -minütige therapeutische Sitzungen. Die NB-Kontroll-Gruppe erhielt keine Therapie.

Die behandelnden Therapeuten waren lizenzierte FachleutemiteinemDoktorgradoderMasterabschluss. Die vier EMDR-Behandler waren ausgebildete und zertifizierte EMDR-Therapeuten, wobei zwei den facilitator-Status innehatten. Die vier BiofeedbackBehandler waren durch das Biofeedback Certification Institute of America ausgebildet und zertifiziert worden. Bei den Biofeedback-Behandlungen kam die bereits vor Ort befindliche Behandlungsausrüstung zum Einsatz. Um eine Befangenheit (bias) der Untersucher auszuschließen, nahm der Testleiter nicht als Therapeut teil, verfügte jedoch über umfangreiches Wissen sowohl in EMDR als auch Biofeedback.

\section{Ergebnisse}

Die Daten wurden einer wechselseitige Varianzanalyse (ANOVA) mit wiederholten Messungen unterzogen, um herauszufinden, ob es einen signifikanten 
Zusammenhang zwischen den Gruppen und den Prä- / Post-Testungen gab. Sofern eine signifikante Wechselwirkung zu erkennen war, wurden paarweise Vergleiche unter Verwendung des NewmanKeuls Tests durchgeführt, um bestimmen zu können, ob ein statistischer Unterschied zwischen den Mittelwerten vorlag. Vergleiche wurden für die Vorbehandlungs-Durchschnittswerte und die durchschnittlichen Veränderungswerte (Vorbehandlungsminus Nachbehandlungs-Punktzahl) durchgeführt. Die klinische Signifikanz (im Sinne von Wirksamkeit, Anm. d. Ü.) wurde für jede Behandlungsmaßnahme durch die Bestimmung des Prozentsatzes der Prä- / Post-Veränderung ermittelt. Zusätzlich wurde sie bestimmt durch die Feststellung des perzentilen Rangs der Prä- / Post-Mittelwerte für das TAI und das STAI unter Verwendung der Norm-Tabellen in jedem Behandlungs-Manual für College-Studentinnen.

\section{STAI-Trait}

Die Ergebnisse der ANOVA zeigten einen signifikanten Zusammenhang zwischen der Kohorten-Zuordnung und den Prä- / Post-Punktzahlen, $F(2,27)=$ 12,97, $p<.001$. Newman-Keuls Tests ließen keinen signifikanten Unterschied zwischen den STAI-T Mittelwerten vor Behandlungsbeginn erkennen. Anschließende Newman-Keuls Analysen für den STAI-T ergaben, dass sowohl EMDR als auch B / SIT größere Veränderungen als NB bewirkten $(p<.05)$. Auch, wenn kein signifikanter Unterschied zwischen den zwei Behandlungs-Kohorten vorlag, veränderte sich die Punktzahl der EMDR-Teilnehmer auf der Normskala von der 93. zur 52. Perzentile, was einen Veränderungswert von 25,7 \% ergibt. Der Perzentil-Wert der B / SIT-Teilnehmer fiel von der 76. auf die 65. Perzentile, was einer Veränderung von 6,7 \% entspricht (siehe Tabellen 1 und 2 und Abbildung 1).

\section{STAI-State}

Die Ergebnisse der ANOVA zeigten einen signifikanten Zusammenhang zwischen der KohortenZuordnung und den Prä- / Post-Tests, $F(2,27)=3,94$, $p<.004$. Newman-Keuls Tests ließen keinen signifikanten Unterschied zwischen den STAI-S Mittelwerten vor Behandlungsbeginn erkennen. Anschließende Newman-Keuls Analysen für den STAI-S ergaben, dass sowohl EMDR als auch B / SIT größere Veränderungen als NB bewirkten $(p<.05)$. Auch, wenn kein signifikanter Unterschied zwischen den zwei Behandlungs-Kohorten vorlag, bewegte sich die Punktzahl der EMDR-Teilnehmer auf der Normskala von der 96. zur 27. Perzentile, was einer Änderung von 45,4 \% entspricht. Der Perzentil-Wert der B / SITTeilnehmer fiel von 66 auf 52, was einer Veränderung von 9,9\% gleichkommt (siehe Tabellen 1 und 2 und Abbildung 2).

\section{TAI-Gesamtwert}

Die Ergebnisse der ANOVA zeigten einen signifikanten Zusammenhang zwischen der KohortenZuordnung und den Prä- / Post-Tests, $F(2,27)=26,30$, $p<.001$. Nachträgliche Newman-Keuls-Tests ließen keinen signifikanten Unterschied zwischen den TAIGesamt-Mittelwerten vor Behandlungsbeginn erkennen, ergaben jedoch, dass sowohl EMDR als auch B / SIT größere Veränderungen als NB bewirkten $(p<.05)$ und dass die EMDR-Mittelwerte signifikant höher waren als bei B / SIT $(p<.05)$. Die Punktzahl der EMDR-Teilnehmer änderte sich auf der Normskala von der 96. zur 30. Perzentile, was einer Veränderung von 47,3\% entspricht. Der Perzentil-Wert der B / SIT-Teilnehmer fiel von 96 auf 84, was einer Veränderung von $16,4 \%$ gleichkommt (siehe Tabellen 1 und 2 und Abbildung 3 ).

\section{TAI-Besorgnis}

Die Ergebnisse der ANOVA zeigten einen signifikanten Zusammenhang zwischen der KohortenZuordnung und den Prä- / Post-Werten, F(2,27) = $17,01, p<.001$. Nachträgliche Newman-Keuls-Tests ließen keinen signifikanten Unterschied zwischen den TAI-W-Mittelwerten vor Behandlungsbeginn erkennen, ergaben jedoch, dass sowohl EMDR als auch B / SIT größere Veränderungen als NB bewirkten $(p<.05)$ und dass der EMDR-Mittelwert signifikant höher lag als bei B / SIT $(p<.05)$. Die Punktzahl der EMDR-Teilnehmer änderte sich auf der Normskala von der 98. zur 33. Perzentile, was eine Veränderung von $51,4 \%$ ergibt. Der Perzentil-Wert der B / SITTeilnehmer fiel von 97 auf 83, was einer Veränderung von 20,8\% entspricht (siehe Tabellen 1 und 2 und Abbildung 4).

\section{TAI-Emotionalität}

Die Ergebnisse der ANOVA zeigten einen signifikanten Zusammenhang zwischen der Kohorten-Zuordnung und den Prä- / Post-Punktzahlen, $F(2,27)=24,52$, $p<.001$. Nachträgliche Newman-Keuls-Tests lieBen keinen signifikanten Unterschied zwischen den TAI-E-Mittelwerten vor Behandlungsbeginn erkennen, ergaben jedoch, dass sowohl EMDR als auch B / SIT größere Veränderungen als NB bewirkten 
TABELLE 1. Mittelwerte (M) und Standardabweichungen (SA) vor Behandlungsbeginn, nach Behandlungsabschluss und Veränderungswerte

\begin{tabular}{|c|c|c|c|c|c|c|c|c|c|}
\hline & \multicolumn{3}{|c|}{ Behandlungsbeginn } & \multicolumn{3}{|c|}{ Behandlungsabschluss } & \multicolumn{3}{|c|}{ Veränderungswerte } \\
\hline & $M$ & $S A$ & Perzentile & $M$ & $S A$ & Perzentile & $M$ & $S A$ & \% Veränderung \\
\hline \multicolumn{10}{|c|}{ TAI-Gesamtwert } \\
\hline EMDR & 64.3 & 10.88 & 96th & 33.9 & 9.7 & 30 th & 30.40 & 7.40 & 47.3 \\
\hline B / SIT & 64.2 & 10.00 & 96th & 53.7 & 13.1 & 84 th & 10.50 & 11.81 & 16.4 \\
\hline NT & 66.8 & 10.70 & 98th & 64.9 & 12.6 & 97 th & 1.90 & 6.93 & 2.8 \\
\hline \multicolumn{10}{|c|}{ TAI-Besorgnis } \\
\hline EMDR & 24.7 & 5.10 & 98th & 12.0 & 3.9 & $33 \mathrm{rd}$ & 12.70 & 3.92 & 51.4 \\
\hline B / SIT & 24.5 & 5.10 & 97th & 19.4 & 6.6 & $83 \mathrm{rd}$ & 5.10 & 5.68 & 20.8 \\
\hline NT & 27.1 & 4.80 & 99th & 25.1 & 5.5 & 98th & 2.00 & 2.40 & 7.4 \\
\hline \multicolumn{10}{|c|}{ TAI-Emotionalität } \\
\hline EMDR & 27.1 & 4.50 & 94th & 14.1 & 3.9 & 26th & 13.00 & 3.265 & 48.0 \\
\hline B / SIT & 26.7 & 4.10 & 93rd & 22.6 & 5.4 & 78th & 4.10 & 5.04 & 15.4 \\
\hline NT & 27.4 & 5.00 & 94 th & 26.6 & 6.0 & 92nd & 0.80 & 3.55 & 2.9 \\
\hline \multicolumn{10}{|l|}{ STAI-Trait } \\
\hline EMDR & 54.1 & 11.50 & $93 \mathrm{rd}$ & 40.2 & 10.1 & 52nd & 13.90 & 7.06 & 25.7 \\
\hline B / SIT & 46.4 & 8.80 & 76th & 43.3 & 8.5 & 65 th & 3.10 & 6.045 & 6.7 \\
\hline NT & 55.2 & 10.90 & 95 th & 55.0 & 11.8 & 94 th & 0.20 & 5.84 & 0.36 \\
\hline \multicolumn{10}{|l|}{ STAI-State } \\
\hline EMDR & 57.1 & 30.60 & 96th & 31.2 & 11.5 & 27 th & 25.90 & 7.96 & 45.4 \\
\hline $\mathrm{B} / \mathrm{SIT}$ & 42.6 & 11.90 & 66 th & 38.4 & 11.6 & 52nd & 4.20 & 10.22 & 9.9 \\
\hline NT & 52.3 & 14.40 & 90th & 49.5 & 15.9 & 85 th & 2.80 & 5.84 & 5.4 \\
\hline \multicolumn{10}{|l|}{ APQ } \\
\hline EMDR & 106.0 & 30.70 & & 44.0 & 31.0 & & 62.00 & 24.77 & 58.5 \\
\hline B / SIT & 95.6 & 25.90 & & 66.2 & 33.0 & & 29.40 & 32.49 & 30.8 \\
\hline NT & 106.4 & 40.20 & & 98.9 & 43.9 & & 7.54 & 21.19 & 7.1 \\
\hline \multicolumn{10}{|l|}{ RBI } \\
\hline EMDR & 21.7 & 4.60 & & 23.8 & 2.7 & & -2.10 & 2.77 & -9.7 \\
\hline B / SIT & 24.2 & 3.90 & & 24.6 & 4.0 & & -0.40 & 3.39 & -1.7 \\
\hline NT & 19.4 & 4.70 & & 19.7 & 4.6 & & -0.30 & 3.46 & -1.5 \\
\hline
\end{tabular}

Anmerkung. Perzentile Ränge wurden abgeleitet von manualisierten Behandlungsnormen für College-Studentinnen. TAI = Test Anxiety Inventory; EMDR = Eye Movement Desensitization and Reprocessing; B/SIT = Biofeedback/Stress Inoculation Training; $\mathrm{NT}=$ keine Behandlung; STAI = Stait-Trait Anxiety Inventory; RBI = Rational Behavior Inventory; APQ = Autonomic Perception Questionnaire.

$(p<.05)$ und dass der EMDR-Mittelwert signifikant höher war als bei B / SIT $(p<.05)$. Die Punktzahl der EMDR-Teilnehmer fiel auf der Normskala von der 94. auf die 26. Perzentile, was einer Veränderung von $48,0 \%$ entspricht. Der Perzentil-Wert der B / SITTeilnehmer fiel von 93 auf 78, was einer Veränderung von $15,4 \%$ gleichkommt (siehe Tabellen 1 und 2 und Abbildung 5).

\section{Rational Behavioral Inventory}

Die Ergebnisse der ANOVA zeigten keinen signifikanten Behandlungs-Effekt und keinen Zusammenhang zwischen der Kohorten-Zuordnung und den Prä- / Post-Werten, $F(2,27)=0,89, p=0,423$. Aufgrund dessen wurde kein Newman-Keuls Test vorgenommen (siehe Tabellen 1 und 2 und Abbildung 6). 
TABELLE 2. Newman-Keuls Analyse: Vergleich der gemittelten Veränderungswerte

\begin{tabular}{lcccc}
\hline & $\begin{array}{c}\text { EMDR }> \\
\text { B } / \text { SIT }\end{array}$ & $\begin{array}{c}\text { EMDR / B / } \\
\text { SIT }>\text { NT }\end{array}$ & $\begin{array}{c}\text { EMDR }> \\
\text { NT }\end{array}$ & $\begin{array}{c}\text { B / SIT }> \\
\text { NT }\end{array}$ \\
\hline TAI & $\mathrm{Y}$ & $\mathrm{Y}$ & $\mathrm{Y}$ & $\mathrm{Y}$ \\
TAI-W & $\mathrm{Y}$ & $\mathrm{Y}$ & $\mathrm{Y}$ & $\mathrm{Y}$ \\
TAI-E & $\mathrm{Y}$ & $\mathrm{Y}$ & $\mathrm{Y}$ & $\mathrm{Y}$ \\
STAI-T & $\mathrm{N}$ & $\mathrm{Y}$ & $\mathrm{Y}$ & $\mathrm{Y}$ \\
STAI-S & $\mathrm{N}$ & $\mathrm{Y}$ & $\mathrm{Y}$ & $\mathrm{Y}$ \\
RBI & $\mathrm{N}$ & $\mathrm{N}$ & $\mathrm{N}$ & $\mathrm{N}$ \\
APQ & $\mathrm{N}$ & $\mathrm{Y}$ & $\mathrm{Y}$ & $\mathrm{Y}$ \\
\hline
\end{tabular}

Anmerkung. $\mathrm{N}=$ kein signifikanter Unterschied zwischen den Mittelwerten; $\mathrm{Y}=$ signifikanter Unterschied zwischen den Mittelwerten bei $p \leq .05$. EMDR $=$ eye movement desensitization and reprocessing; B $/$ SIT $=$ Biofeedback/Stress Inoculation Training; $\mathrm{NT}=$ keine Behandlung (no treatment); TAI = Test Anxiety Inventory; $\mathrm{STAI}=$ State-Trait Anxiety inventory; $\mathrm{RBI}=$ Rational Behavior Inventory; APQ = Autonomic Perception Questionnaire.

\section{Autonomic Perception Questionnaire}

Die Ergebnisse der ANOVAzeigten einen signifikanten Zusammenhang zwischen der Kohorten-Zuordnung und den Prä- / Post-Werten, F(2,27) = 10,62, $p<$ .001. Nachträgliche Newman-Keuls-Tests ließen keinen signifikanten Unterschied zwischen den APQMittelwerten vor Behandlungsbeginn erkennen. Spätere Newman-Keuls-Analysen des APQ zeigten, dass sowohl EMDR als auch B / SIT größere Veränderungen als NB bewirkten $(p<.05)$. Obwohl es keinen signifikanten Unterschied zwischen den

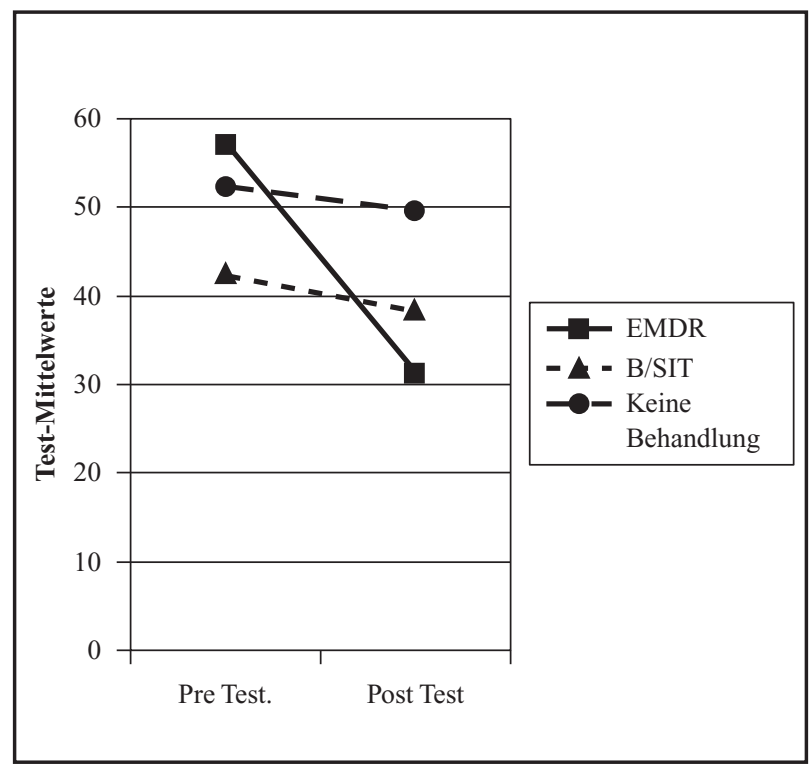

ABBILDUNG 1. Prä-/ Post-Werte gemäßState-Trait-Anxiety Inventory (state).

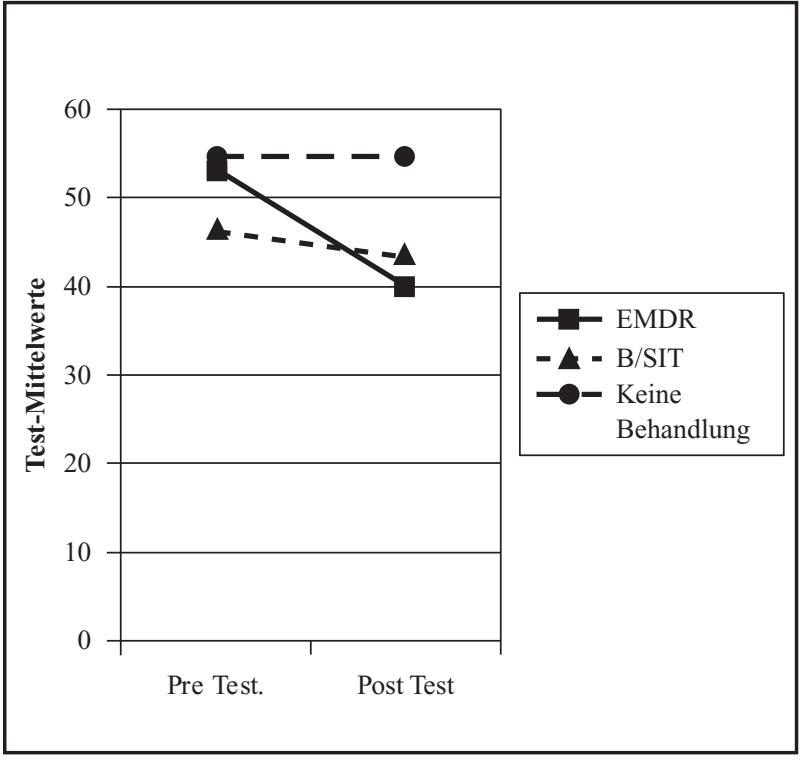

ABBILDUNG 2. Prä- / Post-Werte gemäß State-Trait Anxiety Inventory (trait).

zwei Behandlungsgruppen gab, veränderte sich die Punktzahl von EMDR um 58,5\% und die Punktzahl von B / SIT um 30,8 \% (siehe Tabellen 1 und 2 und Abbildung 7).

\section{Diskussion}

Die Ergebnisse zeigten, dass sowohl EMDR als auch B / SIT Prüfungsangst wirksam reduzierten. Die Anwendung beider Behandlungsmethoden führte in allen Tests (außer dem RBI) zu einer signifikanten Symptomreduktion gegenüber der NB-Kontrollgruppe.

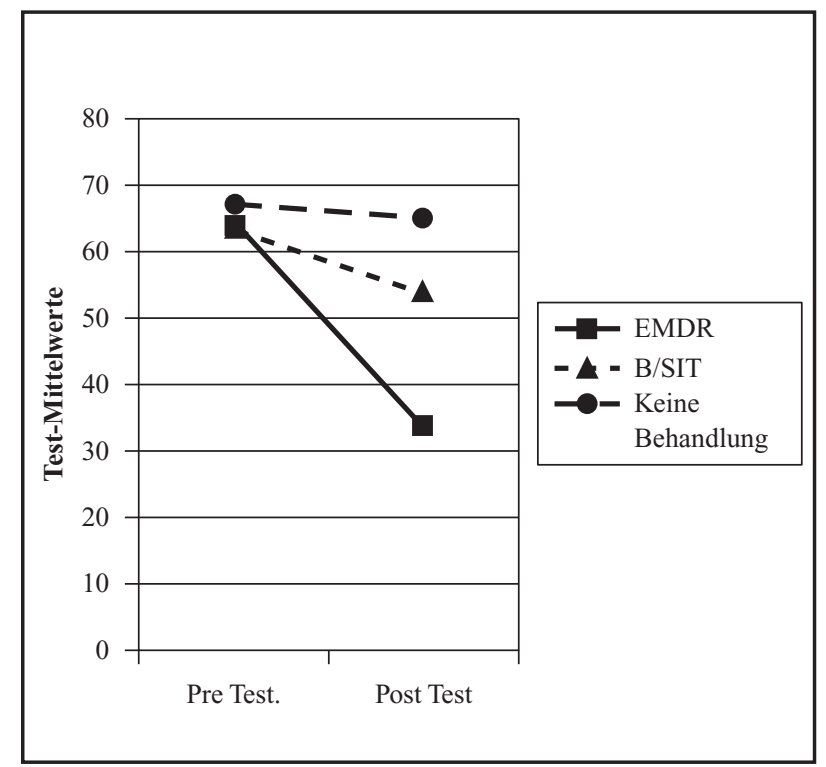

ABBILDUNG 3. Prä- / Post-Werte gemäß Test Anxiety Inventory. 


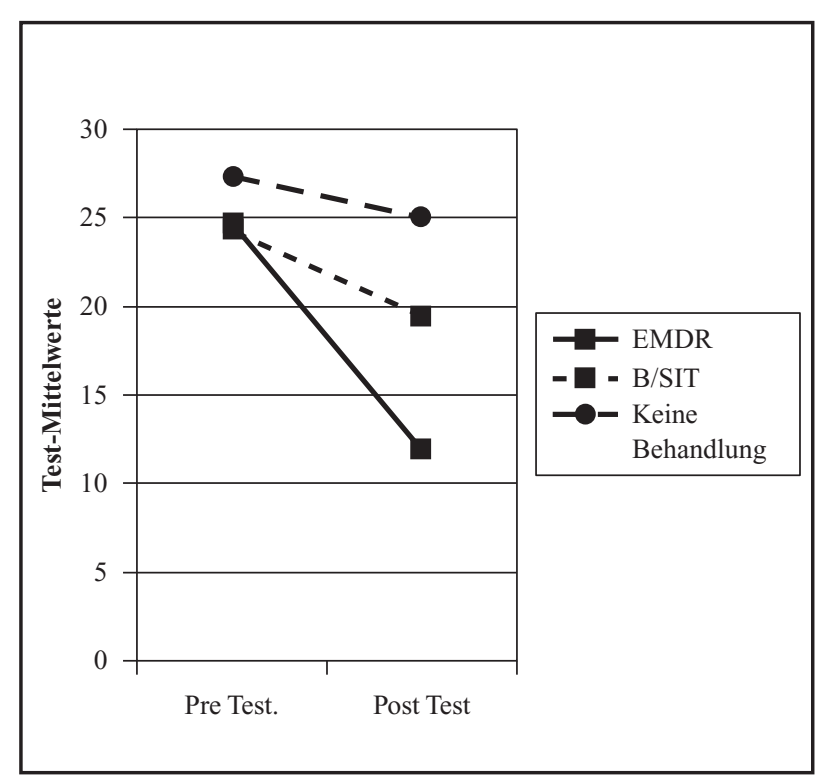

ABBILDUNG 4. Prä- / Post-Werte gemäß Test Anxiety Inventory (Besorgnis).

Die Null-Hypothese, die besagte, dass es keinen Unterschied zwischen EMDR und B / SIT geben würde, wurde bei einem Messwert von $<.05$ für TAI, TAI-E und TAI-W zurückgewiesen. Bei diesen zwei Messungen zeitigte EMDR eine signifikant geringere Symptom-Inzidenz. Es gab keinen signifikanten Unterschied zwischen EMDR und B / SIT auf der APQ, STAI-S oder STAI-T, obwohl EMDR in diesen Messungen durchweg besser abschnitt bzgl. Symptomreduktion als B / SIT (siehe Abbildungen 1, 2 und 7). Auf dem RBI lässt sich für keine der Behandlungen ein Effekt

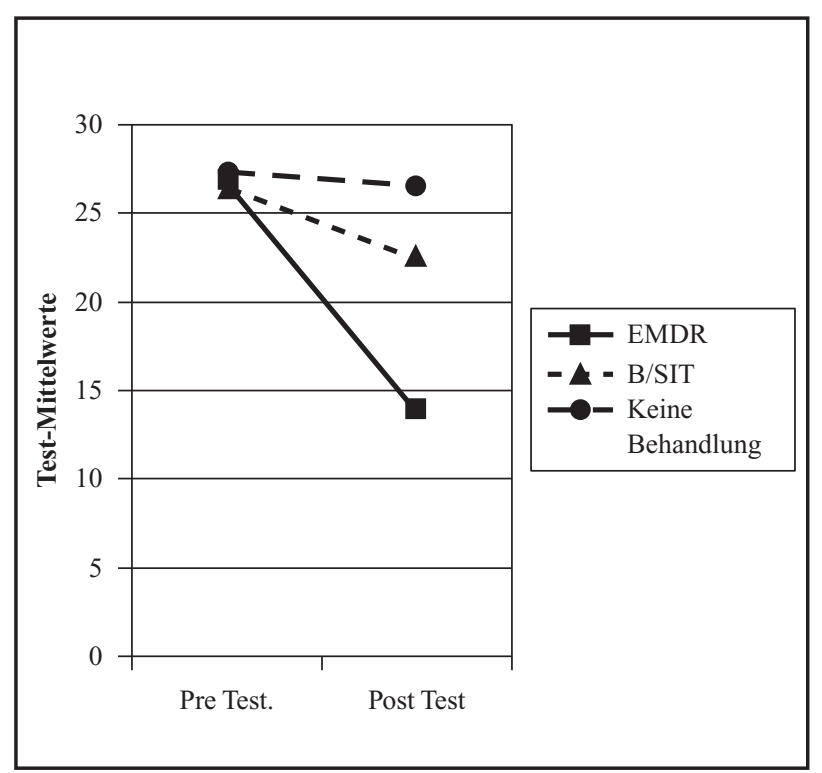

ABBILDUNG 5. Prä- / Post-Werte gemäß Test Anxiety Inventory (Emotionalität).

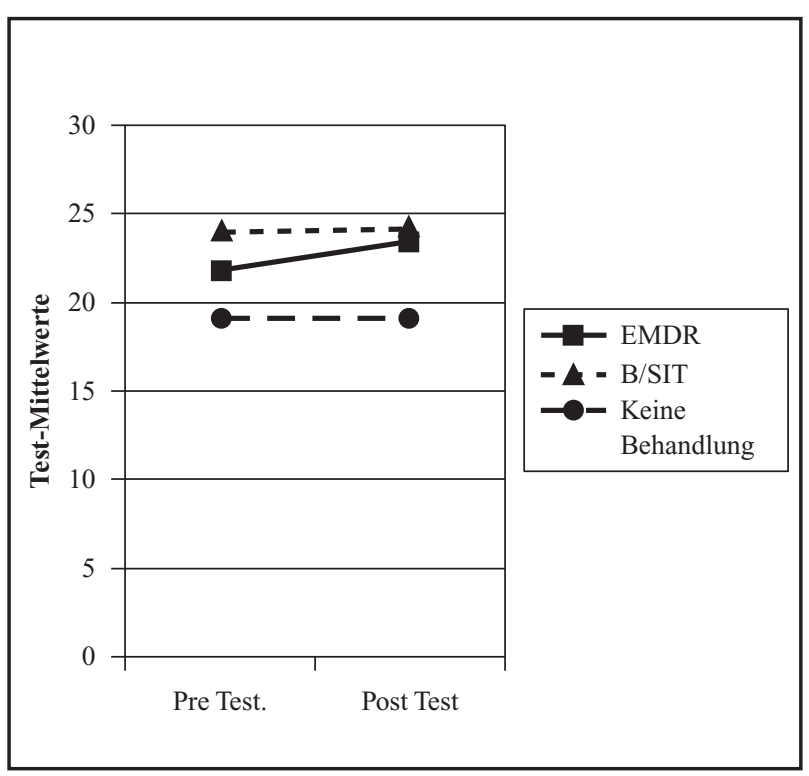

ABBILDUNG 6. Prä- / Post-Werte gemäß Rational Behavioral Inventory

nachweisen. Diese Ergebnisse lassen den vorläufigen Schluss zu, dass Prüfungsangst durch EMDR wirksamer behandelt werden könnte als durch B / SIT.

\section{Prüfungsangst}

Der TAI bewertet Niveaus von Prüfungsangst. In seiner Besorgnis-Subskala, der TAI-W, werden ängstliche Gedanken, Sorgen und die negativen Selbst-Überzeugungen die Prüfungsangst betreffend gemessen. In der Emotionalitäts-Subskala, der TAI-E,

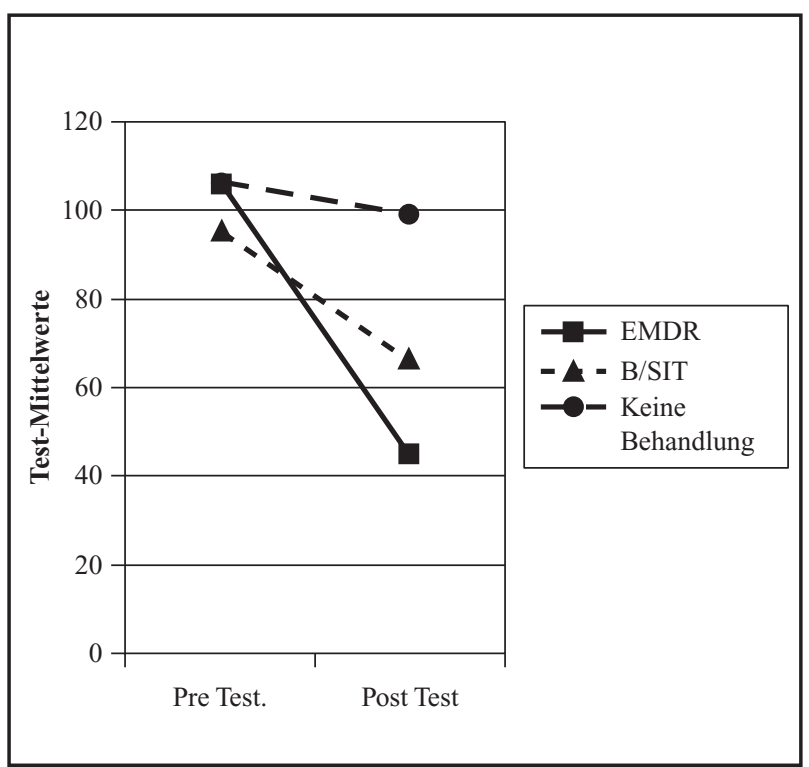

ABBILDUNG 7. Prä- / Post-Werte gemäß Autonomic Perception Questionnaire 
wird die Emotionalität im Zusammenhang mit Prüfungsangst gemessen. Einige der Fragen zielen daher speziell auf das Feststellen der physiologischen Erregung $\mathrm{ab}$, die sich unter anderem in beschleunigtem Herzschlag, Nervosität oder Anspannung äußert.

Obwohl sowohl EMDR als auch B / SIT diese Symptome wirksam reduzierten, bewirkte EMDR jedoch eine signifikant höhere Reduktion von Prüfungsangst als B / SIT. Dies wird in den nachträglichen Newman-Keuls-Analysen aller drei TAI-Skalen deutlich (siehe Abbildungen 3, 4 und 5). Im Ausmaß der Symptom-Reduktion ergaben sich große Unterschiede zwischen B / SIT und EMDR. Zum Beispiel lag die Prä- / Post-Reduktion der TAIW-Punktzahl von EMDR bei 51,4 \% und dagegen bei B / SIT nur bei 21,8 \% (siehe Tabelle 1). Die EMDR-Kohorte zeigte einen Rückgang aller drei Messergebnisse, der mehr als doppelt so hoch war wie der der B / SIT - Behandlungsgruppe. Diese Unterschiede werden auch in der Perzentil-Rangfolge deutlich. Die Analyse der klinischen Effekte zeigte, dass der Perzentil-Wert von EMDR in der BesorgnisSkala von 98 auf 33 fiel, wohingegen der von B / SIT von 97 auf 83 und der von NB von 99 auf 98 fiel. Angesichts der Ergebnisse des TAI scheint EMDR wirksamer in der Behandlung von Prüfungsangst zu sein als B / SIT.

\section{State und Trait Anxiety}

Der STAI misst die Ausprägung von State-Angst (situationsspezifischer, temporärer Angstzustand) und Trait-Angst (persistierender Charakterzug im Sinne einer relativ stabilen Angstanfälligkeit). Da es sich bei Prüfungsangst um eine State-Angst handelt, wurden die Teilnehmer gebeten zu erinnern, wie sie sich während der Testbatterie für die Studie fühlten. Sowohl EMDR als auch B / SIT zeigten im Vergleich $\mathrm{zu}$ NB eine signifikante Reduktion von State- und Trait-Angst. Obwohl auf beiden Skalen kein signifikanter Unterschied zwischen den zwei Behandlungsformen $\mathrm{zu}$ erkennen war, gab es doch einen offensichtlichen Vorteil für EMDR. Beispielsweise ergab sich für EMDR eine Reduktion der Prä- / PostSTAI-S-Mittelwerte von 45,4\% gegenüber 9,9\% für die B / SIT-Kohorte. Für EMDR veränderte sich die perzentile Rangfolge von 96 auf 27 im Vergleich zu B / SIT mit einer Veränderung von 66 auf 52. Die unzureichende statistische Signifikanz mag auf die geringe Gruppenstärke von 10 Teilnehmern pro Gruppe zurückzuführen sein, oder eine Funktion der erheblichen Varianz bei den Behandlungswerten. Dieser Aspekt bedarf weiterer Untersuchung.
Rationalität (Angemessenheit) von Selbst-Überzeugungen

Das RBI misst die Angemessenheit von SelbstÜberzeugungen. Die Analyse ergab keinen Zusammenhang zwischen Vor- und Nachtests und der Art der Behandlungsgruppe. Anscheinend beeinflussten beide Behandlungsmethoden nicht die auf der RBI dargestellten Symptome. Dieser Befund kann darauf zurückzuführen sein, dass eine signifikante Reduktion von irrationalen Überzeugungen im Zusammenhang mit Prüfungsangst aufgrund der Kürze der Behandlungsdauer für beide Behandlungsgruppen nicht realisierbar war. Weitere Untersuchung muss zeigen, ob längere Behandlungsdauer mit EMDR oder B / SIT stärkere Veränderungen in der Angemessenheit persönlicher Überzeugungen in Zusammenhang mit Prüfungsangst bewirken kann.

\section{Erlebte (perceived) physiologische Erregung}

Der APQ misst das Ausmaß der erlebten physiologischen Erregung. Verglichen mit der NB-Kohorte zeigten sowohl EMDR als auch B / SIT eine signifikante Reduktion der Werte für die erlebte physiologische Erregung gemäß APQ. Es gab keinen signifikanten Unterschied zwischen den Behandlungsgruppen, jedoch zeigte die EMDR-Behandlungsgruppe eine höhere Reduktion: 58,5 \% im Mittelwert bei der EMDR-Behandlungsgruppe, 30,8 \% bei der B / SITund $7,1 \%$ bei der NB-Behandlungsgruppe.

Eine der Fragen, der in der Studie nachgegangen wurde, war, ob EMDR vergleichbar ist mit einer anerkannten Behandlungsweise von Prüfungsangst. Prüfungsangst beinhaltet eine Komponente emotionaler oder physiologischer Erregung, die erfolgreich mit Biofeedback behandelt worden ist (Romano \& Cabianca, 1978). EMDR wurde unter anderem deshalb mit Biofeedback verglichen, um herauszufinden, ob es ebenso effektiv physiologische Erregungssymptome reduzieren würde. Die Ergebnisse des APQ zeigen, dass EMDR ebenso effektiv wie Biofeedback oder diesem sogar überlegen ist bei der Behandlung von Symptomen physiologischer Erregung. Sowohl die Ergebnisse des TAI-E als auch des APQ belegen anscheinend die Wirksamkeit von EMDR in der Behandlung von physiologischer Erregung.

\section{Zusammenfassung}

In dieser Studie wurde die Behandlung mit EMDR und B / SIT mit einer Nicht-Behandlungsgruppe (NB) verglichen mit dem Ergebnis, dass beide anscheinend wirksam Prüfungsangst beeinflussen. Insbesondere weisen die signifikant niedrigeren TAI-Punktwerte 
auf eine erhebliche Überlegenheit von EMDR gegenüber B / SIT hin. Bei den Punktwerten des STAI oder APQ gab es keine Unterschiede, obgleich der Veränderungs-Prozentsatz von EMDR durchweg gröBer war als der von B / SIT. Die RBI-Werte zeigten, dass beide Behandlungsmethoden keinen Effekt bzgl. irrationaler Selbst-Überzeugungen hatten. Diese Befunde unterstützen die Verwendung von EMDR in Studenten-Beratungszentren zur Behandlung von Prüfungsangst. Hierbei kann laut dieser Studie erwartet werden, dass die Behandlung mit EMDR Studenten dabei helfen könnte, die negativen Auswirkungen von Prüfungsangst zu überwinden.

\section{Empfehlungen für die zukünftige Forschung}

Besondere Beachtungsollte weiterhin die vergleichende Forschung in Bezug auf die Wirksamkeit verschiedener Behandlungsmethoden finden. Bei Replikation dieser Studie sollte das Forschungsdesign eine größere Stichprobe und einen Vortest beinhalten; ebenso eine einmonatige Behandlung vor den Abschluss-Prüfungen, einen Nachtest unmittelbar vor den Prüfungen gefolgt von einem weiteren Nachtest einen Monat nach den Prüfungen. Weiterhin könnte die Kosten- / Zeitaufwand-Effektivität von EMDR und Biofeedback anhand einer Einzelsitzung untersucht werden. Die Evaluation der therapeutischen Wirksamkeit bzw. des Kostenvorteils von längerer versus kürzerer Behandlunsgdauer könnte ebenfalls Gegenstand einer Untersuchung sein. Auch Längsschnitt-Studien zur Langzeit-Wirkung von EMDR im Vergleich zu B / SIT könnten in Betracht gezogen werden.

Übersetzung von Marlen Lenzen

\section{Fußnoten}

1. Anm. d. Ü.: Im Original: „,Waitlist / delayed treat-

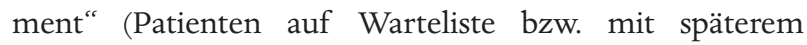
Behandlungsbeginn).

2. Anm. d. Ü.: Darunter versteht man, dass dem Klienten lediglich Informationen zu seiner Störung bereitgestellt werden, aber keine therapeutische Intervention erfolgt.

3. Anm. d. Ü.: State bezeichnet einen vorübergehenden psychisch-organismischen Zustand; trait bezeichnet eine Persönlichkeitseigenschaft im Sinne einer situationsunabhängigen, persistierenden reaktiven Neigung. Da es im Deutschen keine knappe und elegante Entsprechung gibt, verwende ich im Folgenden die englische Nomenklatur.

\section{Literatur}

American Psychiatric Association. (2004). Practice guideline for the treatment of patients with acute stress disorder and posttraumatic stress disorder. Arlington, VA: Author.
Bauman, W., \& Melnyk, W. T. (1994). A controlled comparison of eye movements and finger tapping in the treatment of test anxiety. Journal of Behavior Therapy and Experimental Psychiatry, 25(1), 29-33.

Beidel, D. C., Turner, S. M., \& Dancu, C. V. (1985). Physiological, cognitive and behavioral aspects of social anxiety. Behaviour Research and Therapy, 23(2), 109-117.

Bisson, J., \& Andrew, M. (2007/2009). Psychological treatment of post-traumatic stress disorder (PTSD). Cochrane Database of Systematic Reviews, 3, CD003388.

Borkovec, T. D. (1978). Treatment of general tension: Subjective and physiological effects of progressive relaxation. Journal of Behavior Therapy and Experimental Psychiatry, 25, 29-33.

Bradley, R., Greene, J., Russ, E., Dutra, L., \& Westen, D. (2005). A multidimensional meta-analysis of psychotherapy for PTSD. The American Journal of Psychiatry, 162(2), 214-227.

Carlson, J. G., Chemtob, C. M., Rusnak, K., Hedlund, N. L., \& Muraoka, M. Y. (1998). Eye movement desensitization and reprocessing (EMDR) treatment for combat-related posttraumatic stress disorder. Journal of Traumatic Stress, 11(1), 3-24.

Cassady, J. C., \& Johnson, R. E. (2002). Cognitive test anxiety and academic performance. Contemporary Educational Psychology, 27(2), 270-295.

Counts, D. K., Hollandsworth, J. G., \& Alcorn, J. D. (1978). Use of electromyographic biofeedback and cue-controlled relaxation in the treatment of test anxiety. Journal of Consulting and Clinical Psychology, 46(5), 990-996.

De Jongh, A., \& ten Broeke, E. (2009). EMDR and the anxiety disorders: Exploring the current status. Journal of EMDR Practice and Research, 3(3), 133-140.

Eckman, P. S., \& Shean, G. D. (1997). Habituation of cognitive and physiological arousal and social anxiety. Behaviour Research and Therapy, 35(12), 1113-1121.

Enright, M., Baldo, T. D., \& Wykes, S. D. (2000). The efficacy of eye movement desensitization and reprocessing therapy technique in the treatment of test anxiety of college students. Journal of College Counseling, 3(1), 36-49.

Falsetti, S. (2003). Cognitive-behavioral therapy in the treatment of posttraumatic stress disorder. Primary Psychiatry, 10(5), 78-83.

Gosselin, P., \& Matthews, W. J. (1995). Eye movement desensitization and reprocessing in the treatment of test anxiety: A study of the effects of expectancy and eye movement. Journal of Behavior Therapy and Experimental Psychiatry, 26(4), 331-337.

Hurwitz, L., Kahane, J., \& Mathieson, C. (1986). The effects of EMG biofeedback and progressive muscle relaxation on the reduction of test anxiety. Educational and Psychological Research, 6(4), 291-298.

Kiselica, M., Baker, S., Thomas, R., \& Reedy, S. (1994). Effects of stress inoculation training on anxiety, stress, and academic performance among adolescents. Journal of Counseling Psychology, 41(3), 335-342. 
Lee, C. W., Gavriel, H., Drummond, P., Richards, J., \& Greenwald, R. (2002). Treatment of PTSD: Stress inoculation training with prolonged exposure compared to EMDR. Journal of Clinical Psychology, 58(9), 1071-1089.

Maxfield, L., \& Melnyk, W. (2000). Single session treatment of test anxiety with eye movement desensitization and reprocessing (EMDR). International Journal of Stress Management, 7(2), 87-101.

Meichenbaum, D. (1996). Stress inoculation training for coping with stressors. The Clinical Psychologist, 49, 4-7.

Naveh-Benjamin, M., Lavi, H., McKeachie, W. J., \& Lin, Y. (1997). Individual differences in students' retention of knowledge and conceptual structures learned in university and high school courses: The case of test anxiety. Applied Cognitive Psychology, 11(6), 507-526.

Pluess, M., Conrad, A., \& Wilhelm, F. H. (2009). Muscle tension in generalized anxiety disorder: A critical review of the literature. Journal of Anxiety Disorders, 23(1), $1-11$.

Ratanasiripong, P., Sverduk, K., Hayashino, D., \& Prince, J. (2010). Setting up the next generation biofeedback program for stress and anxiety management for college students: A simple and cost-effective approach. College Student Journal, 44(1), 97-100.

Reed, M., \& Saslow, C. (1980). The effects of relaxation instructions and EMG biofeedback on test anxiety, general anxiety, and locus of control. Journal of Clinical Psychology, 36(3), 683-690.

Rehm, L. P., \& Rokke, P. (1988). Self-management therapies. In K. S. Dobson (Ed.), Handbook of cognitive-behavioral therapies (pp. 136-166). New York: Guilford Press.

Romano, J. L., \& Cabianca, W. A. (1978). EMG biofeedback training versus systematic desensitization for test anxiety reduction. Journal of Counseling Psychology, 25(1), $8-13$.

Rothbaum, B. O., Astin, M. C., \& Marsteller, F. (2005). Prolonged exposure versus eye movement desensitization and reprocessing (EMDR) for PTSD rape victims. Journal of Traumatic Stress, 18(6), 607-616.

Saunders, T., Driskell, J. E., Johnston, J. H., \& Salas, E. (1996). The effect of stress inoculation training on anxiety and performance. Journal of Occupational Health Psychology, 1(2), 170-186.

Schulz, S. M., Alpers, G. W., \& Hoffman, S. G. (2008). Negative self-focused cognitions mediate the effect of trait social anxiety on state anxiety. Behaviour Research and Therapy, 46(4), 438-449.

Schwartz, M. S., \& Andrasik, F. (Eds.). (2003). Biofeedback: A practitioner's guide (3rd ed.). New York: Guilford Press.

Shapiro, F. (2001). Eye movement desensitization and reprocessing: Basic principles, protocols, and procedures (2nd ed.). New York: Guilford Press.
Sheehy, R. S., \& Horan, J. J. (2004). The effects of stressinoculation training for first year law students. International Journal of Stress Management, 11, 41-55.

Shorkey, C. T., \& Whiteman, V. L. (1977). Development of the rational behavior inventory: Initial validity and reliability. Educational and Psychological Measurement, 37, 527-534.

Spielberger, C. D. (1980). Test anxiety inventory manual. Palo Alto, CA: Mind Garden.

Spielberger, C. D. (1983). State-trait anxiety inventory for adults. Palo Alto, CA: Mind Garden.

Spielberger, C. D. (2008). State-trait anxiety inventory for adults. Retrieved December 15, 2011, from http: / / www .mindgarden.com/products/staisad.htm

Spies, G. (1979). Desensitization of test anxiety: Hypnosis compared with biofeedback. American Journal of Clinical Hypnosis, 22(2), 108-111.

Stevens, M., \& Florell, D. (1999). EMDR as a treatment for test anxiety. Imagination, Cognition, and Personality, 18(4), 285-296.

Thyer, B., Papsdorf, J., Himle, D., McCann, B., Caldwell, S., \& Wickert, M. (1981). In vivo distraction-coping in the treatment of test anxiety. Journal of Behavioral Clinical Psychology, 3(4), 754-764.

U.S. Department of Veterans Affairs, \& U.S. Department of Defense (2004/2010). VA/DoD clinical practice guideline for the management of post-traumatic stress. Washington, DC: Author.

Van der Kolk, B., Spinazzola, J., Blaustein, M., Hopper, J. Hopper, E., Korn, D., \& Simpson, W. (2007). A randomized clinical trial of EMDR, fluoxetine and pill placebo in the treatment of PTSD: Treatment effects and long-term maintenance. Journal of Clinical Psychiatry, 68, 37-46.

Danksagung. Unsere geschätzte Kollegin und Co-Autorin Dr. Rosemary Cook-Vienot verstarb unerwartet am 29. Dezember 2008. Dieser Artikel ist ihrem Andenken, ihrem Leben und ihrer Arbeit gewidmet. Sie war meine Freundin, meine Mentorin und Vertraute. Ich weiß, dass es ihr viel bedeuten würde, ihre Tochter Aimee Atkinson, die vor ihr verstarb, für ihre Arbeit als Forschungs-Assistentin und Koordinatorin zu würdigen. Ebenso würde Dr. Vienot die EMDR- und Biofeedback-Behandlungsteams gewürdigt wissen und ihnen danken wollen: David J. Ogren, Marcia Cotton, Vivian Freytag, Alan Schneider, Helen Alexander, Victoria Harrison und Vicki Jones. Auch Katalin Grajzel möchte ich meinen Dank aussprechen für ihre Forschungsund Redaktions-Assistenz.

Zuschriften zu diesem Artikel bitte an: Raymond J. Taylor, Counseling Services of Houston, Texas, P.O. Box 9023, Pueblo, CO 81008. E-mail: rtmtrt@aol.com 